\title{
Advanced glycation end products induce glomerular endothelial cell hyperpermeability by upregulating matrix metalloproteinase activity
}

\author{
PENGLI LUO ${ }^{1 *}$, HUI PENG ${ }^{2 *}$, CANMING LI $^{2}$, ZENGCHUN YE ${ }^{2}$, HUA TANG $^{2}$, \\ YING TANG ${ }^{3},{\text { CAILIAN } \mathrm{CHEN}^{2} \text { and TANQI LOU }}^{2}$ \\ ${ }^{1}$ Department of Nephrology, Affiliated Hospital of Qinghai University, Xining, Qinghai 810001; \\ ${ }^{2}$ Department of Nephrology, The Third Affiliated Hospital of Sun Yat-Sen University, Guangzhou, Guangdong 510660; \\ ${ }^{3}$ Department of Nephrology, The Second Affiliated Hospital of Sun Yat-Sen University, \\ Guangzhou, Guangdong 510000, P.R. China
}

Received February 15, 2014; Accepted November 19, 2014

DOI: $10.3892 / \mathrm{mmr} .2015 .3269$

\begin{abstract}
The present study aimed to investigate the effects of advanced glycation end-products (AGEs) on the permeability of glomerular endothelial cells (GEnCs) and determine whether enhanced permeability was due to degradation of tight junction (TJ) complexes by matrix metalloproteinases (MMPs). Cultured monolayers of GEnCs were exposed to AGEs at different doses and treatment durations in the presence or absence of the organic MMP-2/9 inhibitor (2R)-2-((4-biphenyl sulfony-1) amino)-3-phenylproprionic acid) (BiPs). Expression of the TJ proteins occludin and claudin-5 was determined by western blot analysis and immunofluorescence, while the permeability of the GEnCs was measured using transendothelial electrical resistance and by diffusion of $4 \mathrm{kDa}$ fluorescein isothiocyanate (FITC)-dextran. The activities of MMP-2 and MMP-9 were assayed using gelatin zymography. The results indicated that AGE-treated cultures significantly reduced occludin and claudin-5 immunoreactivity. Similarly, the surface expression of these proteins was significantly reduced and rows of TJs which normally connect endothelial cells became discontinuous or fractured following AGE exposure. Disruption of TJs was accompanied by significantly reduced transendothelial resistance and hyperpermeability to FITC-dextran. Treatment with AGEs evoked a dose- and time-dependent upregulation of MMP-2 and MMP-9. However, co-administration of AGEs and BiPS,
\end{abstract}

Correspondence to: Dr Tanqi Luo, Department of Nephrology, The Third Affiliated Hospital of Sun Yat-Sen University, 600 Tianhe Road, Guangzhou, Guangdong 510660, P.R. China E-mail: luotanqidoctor@126.com

\section{*Contributed equally}

Key words: diabetic nephropathy, glomerular endothelial cells, advanced glycation end products, matrix metalloproteinase, tight junction an inhibitor of MMP-2/MMP-9, inhibited the downregulation of occludin and claudin-5, with a concomitant reversal of GEnC monolayer hyperpermeability. In conclusion, AGEs promoted glomerular hyperpermeability in vitro by the MMP-mediated disruption of TJs. Chronic elevation of endothelial cell AGEs in diabetes mellitus may contribute to glomerular hyperpermeability by inducing the overexpression of MMPs, which degrade TJs, leading to proteinuria.

\section{Introduction}

Hyperpermeability of the microvasculature is one of the most damaging pathological features of diabetes mellitus (1). A number of mechanisms have been proposed to explain endothelial hyperpermeability, including hemodynamic changes (2), oxidative stress (3) and the accelerated production of kinins, histamine or serotonin $(4,5)$.

Advanced glycation end products (AGEs) may also increase vascular permeability $(6,7)$, possibly by activating AGE receptors (RAGEs), which are expressed by endothelial cells. AGEs are formed through nonenzymatic reactions between sugars and amine residues on proteins, lipids and nucleic acids (8). In diabetes, AGEs are known to accumulate in the microvasculature of the kidney and, therefore, may contribute to the pathophysiology of diabetes $(8,9)$. Dynamic regulation of glomerular endothelial cell (GEnC) permeability is pivotal for maintaining the selectivity of glomerular filtration and for preventing proteinuria (10). Indeed, clinical studies have revealed a correlation between serum AGEs and the progression of microalbuminuria (11), suggesting that AGEs may induce pathological increases in glomerular permeability in diabetes mellitus. The impact of AGEs on the pathophysiology of diabetic nephropathy remains to be elucidated.

Matrix metalloproteinases (MMPs) are a family of zinc-dependent proteinases that degrade the extracellular matrix (12). The concentrations and activities of MMP-2 and MMP-9 are increased by high glucose in vivo (13) and in vitro (14). Furthermore, the level of MMP activity is associated with the degree of albuminuria (15-19), suggesting an 
important role of MMP-2/9 in the pathogenesis of diabetic nephropathy. The elevated expression of MMP-2/9 in diabetes may facilitate an increase in vascular permeability by degrading tight junction (TJ) proteins with concomitant disruption of the TJ complex (13).

Enhanced GEnC permeability by the MMP-mediated degradation of TJ proteins has not been demonstrated previously. The present study examined the activity of MMP-2 and MMP-9 in the GEnCs following exposure to exogenous AGEs and the correlation between MMP activity in response to the changes in GEnC's permeability and TJ protein expression. The results indicated a possible role of MMP-mediated TJ proteolysis in diabetic kidney dysfunction.

\section{Materials and methods}

Chemicals and antibodies. AGEs conjugated to bovine serum albumin (BSA) and Calbiochem ${ }^{\circledR}$ MMP-2/MMP-9 Inhibitor II (BiPS) were purchased from Merck KGaA (Darmstadt, Germany). Antibodies to occludin, claudin-5 and Alexa fluor ${ }^{\circledR} 546$ were purchased from Invitrogen Life Technologies (Carlsbad, CA, USA) and GAPDH was purchased from Proteintech Group, Inc. (Chicago, IL, USA). Dylight ${ }^{\circledR} 488$ was purchased from Multiscience Biotech Co., Ltd (Hangzhou, China). Millicell ${ }^{\circledR}$-ERS was purchased from Millipore (Bedford, MA, USA). Fluorescein isothiocyanate (FITC)-dextran was purchased from Sigma-Aldrich (St. Louis, MO, USA).

Cell culture. GEnC cells were purchased from ATCC (Boulevard, MA, USA). Rat GEnC cultures were established and characterized as previously described (20). Briefly, GEnCs were grown in RPMI-1640 medium (Gibco-BRL, Carlsbad, CA, USA) supplemented with $10 \%$ fetal bovine serum (Gibco-BRL) and 10\% NuSerum (Sigma-Aldrich) in a cell incubator at $37^{\circ} \mathrm{C}$ under $5 \% \mathrm{CO}_{2}$. When the cells reached $75-80 \%$ confluence, they were incubated in low-serum medium and treated with AGEs at different doses (20, 40 and $80 \mu \mathrm{l})$ and for various durations (6, 12 and $24 \mathrm{~h})$. In certain experiments, the MMP2/9 inhibitor BiPS $(50 \mu \mathrm{M})$ was also added. The cells and medium were collected $24 \mathrm{~h}$ after the initial treatment.

Western blot analysis. The endothelial cells were treated with lysis buffer and centrifuged at $15,000 \mathrm{x} \mathrm{g}$ for $10 \mathrm{~min}$ at $4^{\circ} \mathrm{C}$. The supernatant was collected for western blotting or stored at $-80^{\circ} \mathrm{C}$ for future analysis. Equal quantities of protein were loaded onto a gel for $15 \%$ SDS-PAGE (Sigma-Aldrich) in order to separate the proteins. Separated proteins were transferred onto polyvinylidene difluoride membranes (Millipore, Lyon, France) and incubated with mouse anti-claudin-5 monoclonal antibody (1:3,000; Santa Cruz Biotechnology, Inc., Dallas, TX, USA) and mouse anti-Occuludin monoclonal antibody (1:2,000; Santa Cruz Biotechnology, Inc.) overnight at $4^{\circ} \mathrm{C}$. Following washing with phosphate-buffered saline (PBS), the appropriate horseradish peroxidase-conjugated rabbit anti-mouse polyclonal secondary antibody $(1: 1,000$; Santa Cruz Biotechnology, Inc.,) was added for $1 \mathrm{~h}$ at room temperature. Protein immunoreactivity was detected using the enhanced chemiluminescence reaction kit (ECL; Pierce, WI, USA), with the Tanon-4200 chemiluminescence reaction detection system (Tiangen Biot Co. Ltd, Beijing China). Band intensities were quantified by Quantity One 2.0 software (Bio-Rad, Hercules, CA, USA).

Transendothelial electrical resistance (TEER) and paracellular permeability assays. The electrical resistance across the confluent cell monolayer, TEER, was measured using the Millicell ${ }^{\circledR}$-ERS system (Millipore) according to the manufacturer's instructions. Briefly, the cells were grown to post-confluence on transwell filters (Corning Costar ${ }^{\circledR}$, Inc., Union City, CA, USA) and treated with AGEs or AGEs + BiPS. The shorter electrode was placed within the Millicell culture plate insert and the longer electrode was placed in the outer well. The resistance of a culture or cell free plate (blank) was measured at three time-points from different points across the inner and outer wells until a stable value was measured each time. The resistance of the blank was subtracted from that measured with endothelial cells (net resistance). The unit area TEER $\left(\mathrm{K} \Omega \cdot \mathrm{cm}^{2}\right)$ was calculated by multiplying the net resistance by the area of the culture plate insert.

For the FITC-dextran flux assays, the cells were grown to post-confluence on transwell filters and $20 \mu \mathrm{l}$ FITC-dextran solution was added to the inner well insert at a final concentration of $1 \mathrm{mg} / \mathrm{ml}$. The medium was collected from the outer well, which was continuous with the basolateral compartment after $24 \mathrm{~h}$. The quantity of diffused FITC-dextran was read using a fluorometer (SpectraMax M5, Molecular Devices, Tokyo, Japan) at wavelengths of $520 \mathrm{~nm}$ emission and $485 \mathrm{~nm}$ excitation.

Immunofluorescence staining. The endothelial cells were grown on glass cover slips to 70-80\% confluence and then cultured in AGEs with or without BiPS for $24 \mathrm{~h}$. The treated cells were fixed in 4\% formaldehyde solution (Sigma-Aldrich) for $10 \mathrm{~min}$, washed in PBS, permeabilized in 0.2\% Triton X-100 (Sigma-A ldrich) plus PBS, blocked with 5\% goat serum for $30 \mathrm{~min}$ and incubated with rabbit anti-claudin-5 polyclonal antibody (1:100; Santa Cruz Biotechnology, Inc.) and mouse anti-Occuludin monoclonal antibody (1:500; Santa Cruz Biotechnology, Inc.) overnight at $4^{\circ} \mathrm{C}$ in humidified chambers. The fixed cells were then probed with a fluorophore-tagged secondary antibody (1:500) for $1 \mathrm{~h}$ at room temperature. The slides were washed in PBS and visualized using a laser scanning confocal microscope (Zeiss LSM510 Meta; Carl Zeiss, Stuttgart, Germany).

Gelatin zymography. To assess gelatinase activity, the cell medium was collected and centrifuged at $12,000 \mathrm{x} \mathrm{g}$ for $10 \mathrm{~min}$ at $4^{\circ} \mathrm{C}$. The supernatant was collected and concentrated 20-fold using Amicon Centricon centrifugal filters (Millipore). The concentrated media was separated electrophoretically on $10 \%$ polyacrylamide gels containing $0.1 \%$ gelatin. Following electrophoresis, gels were washed for $90 \mathrm{~min}$ in $2.5 \%(\mathrm{v} / \mathrm{v})$ Triton X-100 and incubated in enzyme buffer containing $50 \mathrm{mM}$ Tris-HCl, (pH 7.5; Sigma-Aldrich), $150 \mathrm{mM} \mathrm{NaCl}$, $5 \mathrm{mM} \mathrm{CaCl}_{2}$ and $1 \mathrm{M} \mathrm{ZnCl}_{2}$ for $40 \mathrm{~h}$ with gentle agitation at $37^{\circ} \mathrm{C}$. The gel was then stained with $0.2 \%$ Coomassie brilliant blue R-250 (Sigma-Aldrich) in a mixture of methanol, acetic acid and water (2:1:7) for $1 \mathrm{~h}$ and then destained in destaining solution. The band density was quantified by Quantity One 2.0 software (Bio-Rad) following negative image reversal. 

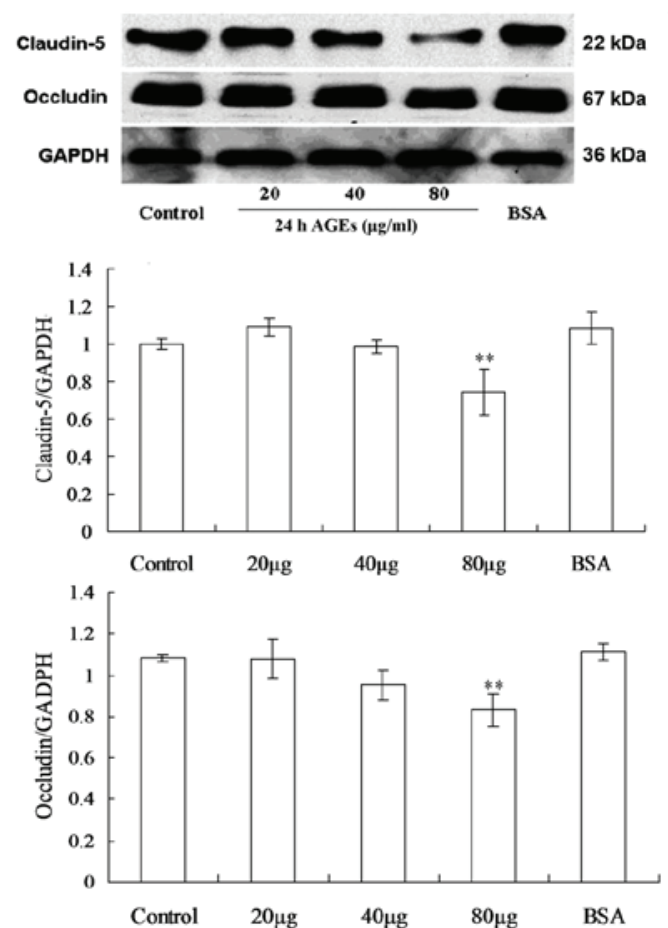

B
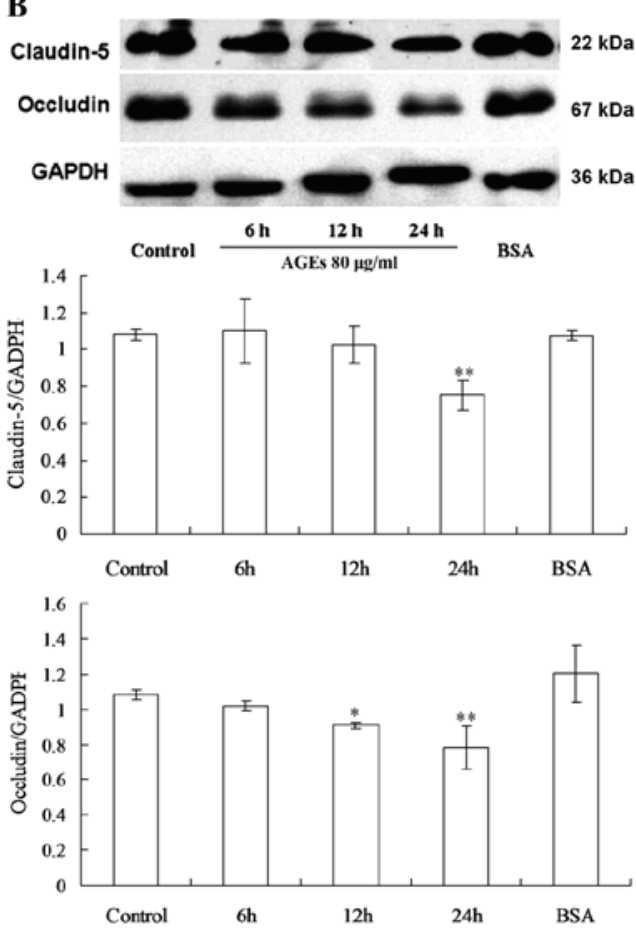

Figure 1. Dose-response and time-course study of AGEs on the expression of occludin and claudin-5 in GEnCs. (A) Expression levels of occludin and claudin-5 decreased markedly following exposure to $80 \mu \mathrm{g} / \mathrm{ml}$ AGEs over $24 \mathrm{~h}$, while exposure to $40 \mu \mathrm{g} / \mathrm{ml}$ AGEs or less did not cause significant changes. (B) GEnCs were treated with AGEs for varying time periods, the expression of occludin began to decrease after $12 \mathrm{~h}$ and reached the lowest level $24 \mathrm{~h}$ after exposure to $80 \mu \mathrm{g} / \mathrm{ml}$ AGEs. Claudin-5 did not decrease significantly unless treated for the full $24 \mathrm{~h}$. ${ }^{*} \mathrm{P}<0.05$, vs. control ${ }^{* *} \mathrm{P}<0.01$, vs. control. AGEs, advanced glycation end-products; GEnCs, glomerular endothelial cells; BSA, bovine serum albumin.

A
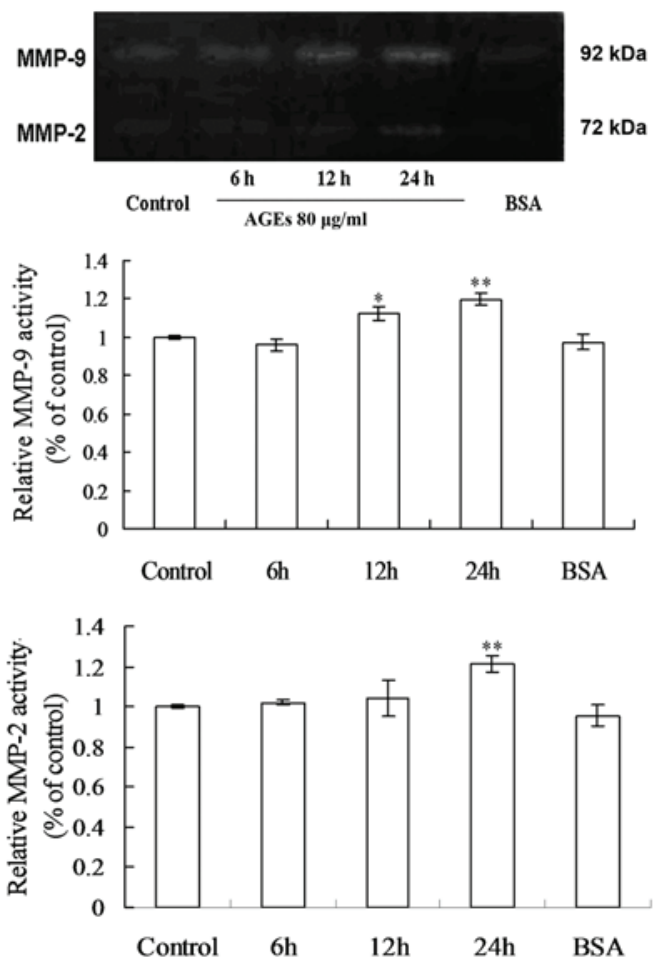

B
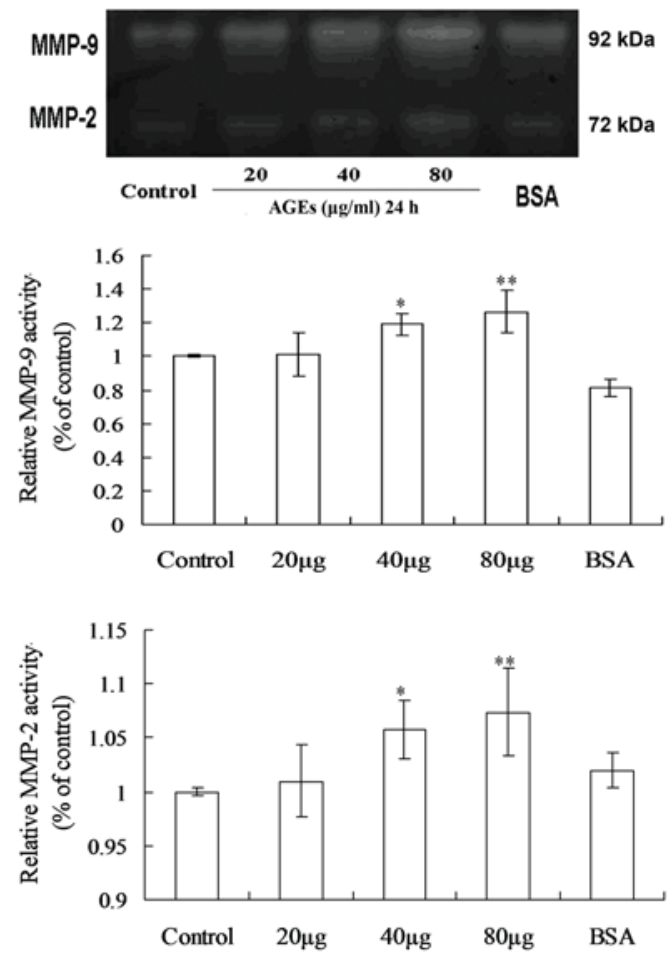

Figure 2. Effect of AGEs on activity of MMP-2 and MMP-9 in a time and dose response analysis in GEnCs. (A) Time course study in which the activity of MMP-9 began increasing after $12 \mathrm{~h}$ and peaked $24 \mathrm{~h}$ after exposure to $80 \mu \mathrm{g} / \mathrm{ml}$ AGEs, while the maximal induction of MMP- 2 activity occurred at $24 \mathrm{~h}$ at the same concentration of AGEs. (B) Dose-response analysis results revealed that proteolytic activities of MMP-2 and MMP-9 were significantly higher following incubation with $40 \mu \mathrm{g} / \mathrm{ml}$ and $80 \mu \mathrm{g} / \mathrm{ml}$ AGEs for $24 \mathrm{~h}$. "P<0.05, vs. control, ${ }^{* * *} \mathrm{P}<0.01$ vs. control. AGEs, advanced glycation end-products; GEnCs, glomerular endothelial cells; BSA, bovine serum albumin; MMP, matrix metalloproteinase. 


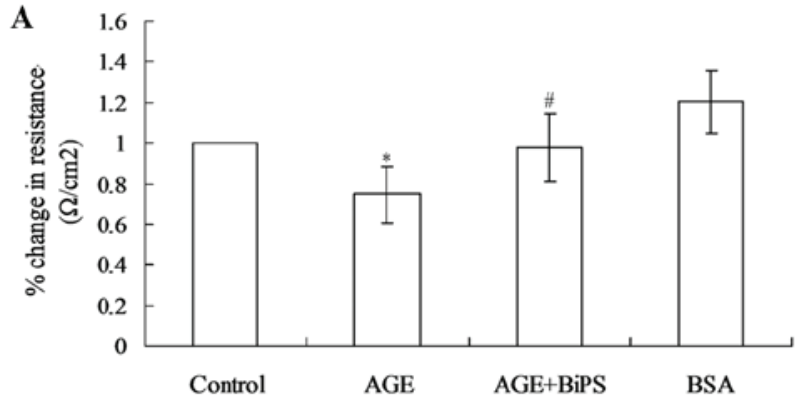

B

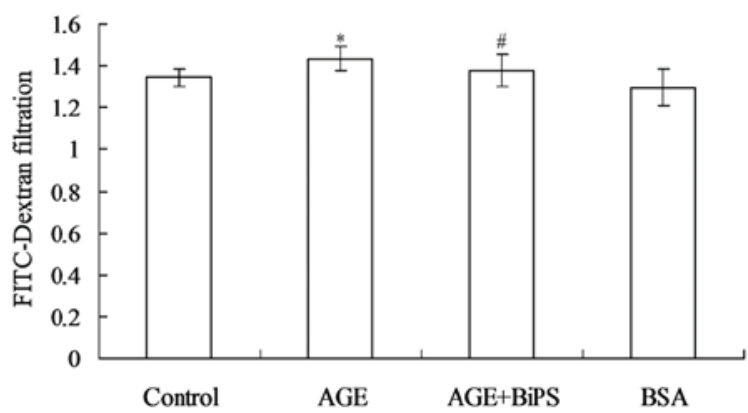

Figure 3. AGE-induced increases in GEnCs permeability and inhibition by BiPS. (A) Exposure of GEnCs to AGEs ( $80 \mu \mathrm{g} / \mathrm{ml})$ significantly decreased the transendothelial electrical resistance levels and (B) increased FITC-dextran filtration. However, co-treatment of cells with BiPS (50 $\mu \mathrm{M})$ significantly attenuated AGEs-induced GEnC hyperpermeability. BSA alone did not alter basal permeability of GEnCs. ${ }^{*} \mathrm{P}<0.05$ vs. control, ${ }^{*} \mathrm{P}<0.05$ vs. AGEs group. AGEs, advanced glycation end-products; GEnCs, glomerular endothelial cells; BSA, bovine serum albumin; MMP, matrix metalloproteinase; FITC, fluorescein isothiocyanate; BiPS, MMP-2/MMP-9 inhibitor II.

Statistical analysis. All the experiments were performed at least three times. The results are presented as the mean \pm standard deviation of independent experiments. Statistically significant differences between treatment groups were determined by one-way analysis of variance using SPSS 19.0 software (International Business Machines, Armonk, NY, USA). $\mathrm{P}<0.05$ was considered to indicate a statistically significant difference.

\section{Results}

Effects of AGEs on the expression of occludin and claudin-5 in the GEnCs. Elevated serum AGE levels have been linked to hyperpermeability of the microvasculature in diabetes mellitus. The altered expression of TJ proteins by endothelial cells may induce these permeability changes; therefore, the expression levels of the TJ proteins occludin and claudin-5 following AGEs exposure at different doses and treatment time periods were examined (Fig. 1). In a dose-response study, GEnCs were exposed to various concentrations of AGEs for $24 \mathrm{~h}$ and the total content of occludin and claudin-5 in the cell lysate was measured by western blot analysis as described previously. The results revealed that exposure of GEnCs to $80 \mu \mathrm{g} / \mathrm{ml}$ AGEs caused a significant decrease of occludin and claudin-5, while exposure to $40 \mu \mathrm{g} / \mathrm{ml}$ or less of AGEs did not cause significant changes (Fig. 1A). To examine the effect of treatment duration, endothelial cells were exposed to $80 \mu \mathrm{g} / \mathrm{ml}$ AGEs for 6,12 , or $24 \mathrm{~h}$. The expression of occludin began to decrease after $12 \mathrm{~h}$ and reached the lowest level after $24 \mathrm{~h}$,

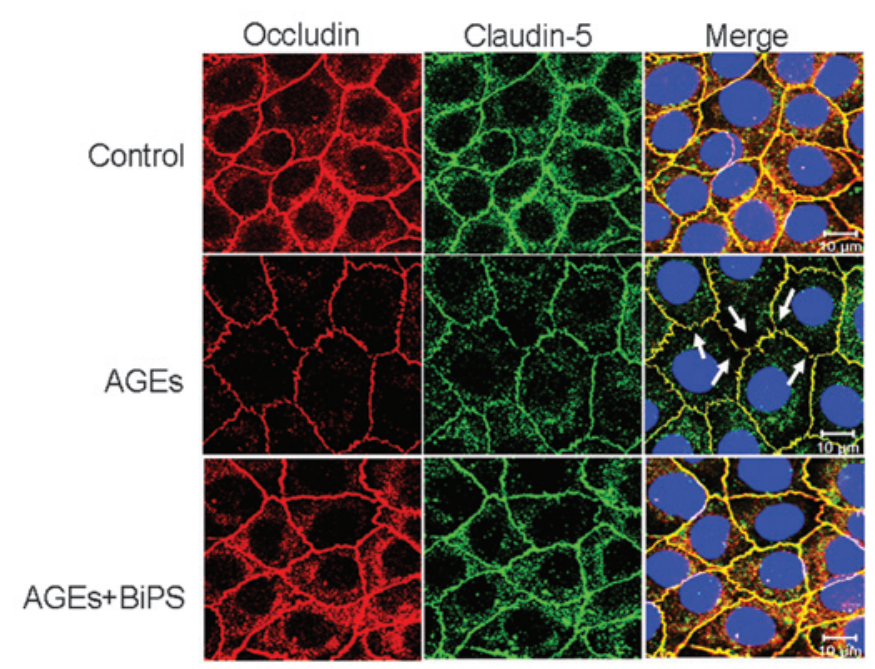

Figure 4. BiPS inhibits the disruption of tight junctions between cells induced by AGEs. Immunofluorescence microscopy demonstrated occludin and claudin-5 staining along the cellular borders, with a smooth, continuous line that encircled the cell in GEnCs in the control group (scale bar, $10 \mu \mathrm{m}$; arrows indicate tight junctions). Incubation of GEnCs with AGEs $(80 \mu \mathrm{g} / \mathrm{ml})$ resulted in decreased intensity of occludin and claudin-5 staining in the cell membrane and the appearance of immunofluorescence staining became discontinuous or fractured at the same time. BiPS $(50 \mu \mathrm{M})$ markedly attenuated AGE-induced alterations in occludin and claudin-5. BiPS, MMP-2/MMP-9 inhibitor II; AGEs, advanced glycation end-products; GEnCs, glomerular endothelial cells.

while claudin-5 did not decrease significantly unless treated for the full $24 \mathrm{~h}$. (Fig. 1B).

Effects of AGEs on the activities of MMP-2 and MMP-9 in the GEnCs. The effects of AGEs on MMP-2 and MMP-9 activity were investigated using gelatin zymography analysis at different doses and durations (Fig. 2). In the time course studies, the results demonstrated that when endothelial cells were exposed to $80 \mu \mathrm{g} / \mathrm{ml}$ AGEs for 6,12 and $24 \mathrm{~h}$, there were significant increases in MMP-9 activity after $12 \mathrm{~h}$ exposure and the activities peaked when treatment was extended to $24 \mathrm{~h}$, while the maximal induction of MMP-2 activity occurred at $24 \mathrm{~h}$ at the same concentration of AGEs (Fig. 2A). The dose-response analysis results demonstrated that the proteolytic activities of MMP-2 and MMP-9 were significantly higher at $40 \mu \mathrm{g} / \mathrm{ml}$ and $80 \mu \mathrm{g} / \mathrm{ml}$ AGEs for $24 \mathrm{~h}$ (Fig. 2B).

GEnC permeability increases in response to treatment with AGEs and is inhibited by BiPS. To assess the effect of AGE treatment on GEnC permeability, the TEER of the confluent GEnC monolayers was measured. After $24 \mathrm{~h}$ exposure to $80 \mu \mathrm{g} / \mathrm{ml}$ AGEs, the GEnCs exhibited a significant decrease in TEER (Fig. 3A). The transendothelial diffusion of FITC-dextran (4 kDa) after $24 \mathrm{~h}$ stimulation with AGEs was also examined, as this molecule may diffuse across the paracellular spaces if endothelial TJs are disrupted. The results of FITC-dextran filtration measurements mirrored the TEER results of the present study; FITC-dextran translocation across the GEnC monolayer was higher in the AGE-treated cultures than that in untreated cultures and BSA did not alter basal permeability of GEnCs (Fig. 3B). To determine the effect of MMP-2 and MMP-9 on AGE-induced GEnC hyperpermeability, BiPS, an inhibitor 

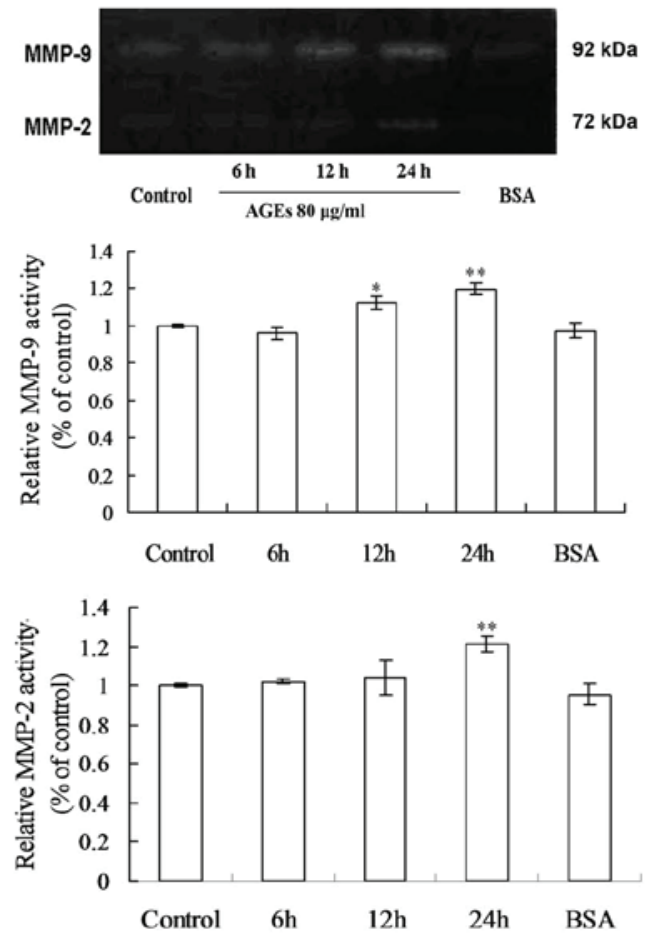

B
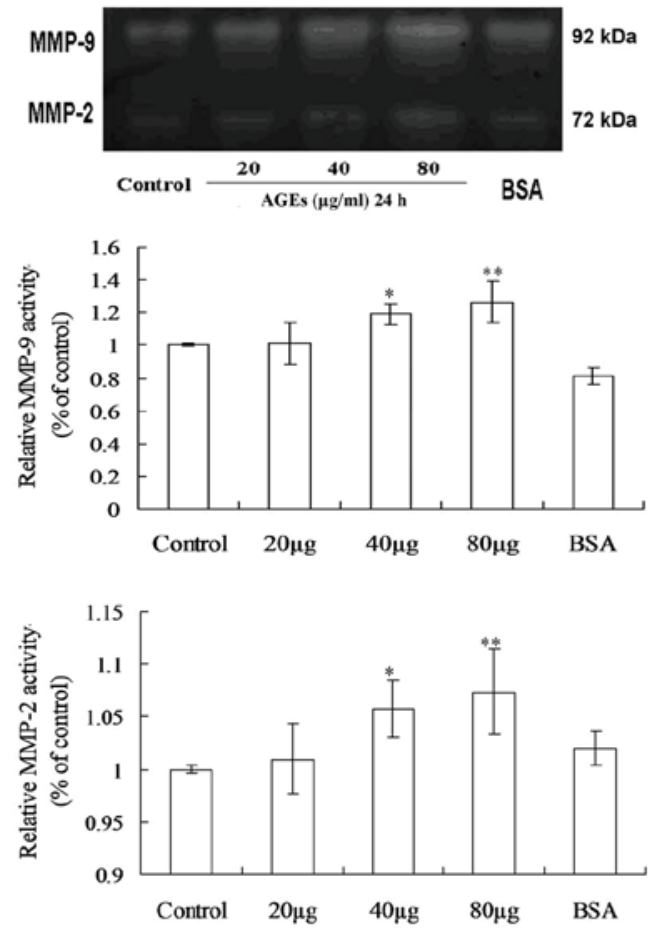

Figure 5. BiPS inhibits the effect of AGEs on expression of occludin, claudin-5 and the activity of MMP-2/9. Expression of occludin and claudin-5 were decreased when GEnCs were exposed to AGEs $(80 \mu \mathrm{g} / \mathrm{ml})$. (A) Downregulation of occludin and claudin-5 expression was reversed by the addition of BiPS (50 $\mu \mathrm{M})$. (B) MMP-2/9 activity was elevated when GEnCs exposed to AGEs. Co-administration with BiPS reversed this AGE-induced increase in MMP-2/9 activity. ${ }^{*} \mathrm{P}<0.05$ vs. control, ${ }^{* *} \mathrm{P}<0.01$ vs. control; ${ }^{\#} \mathrm{P}<0.05$ vs. AGEs group, ${ }^{\# \#} \mathrm{P}<0.01$ vs. AGEs group. AGEs, advanced glycation end-products; GEnCs, glomerular endothelial cells; BSA, bovine serum albumin; MMP, matrix metalloproteinase; BiPS, MMP-2/MMP-9 inhibitor II

of MMP-2/MMP-9, was co-administered with the AGEs. The results revealed that TEER levels and FITC-dextran filtration did not change significantly when the AGE-stimulated cells were co-treated with BiPS compared with those of the control group. These findings supported the role of MMP-2 and MMP-9 as key mediators of AGE-induced hyperpermeability in GEnCs.

BiPS inhibits the disruption of TJs between cells induced by AGEs. To examine molecular changes in the TJs following AGE stimulation, the surface expression levels of occludin and claudin-5 were measured using immunofluorescence (Fig. 4). In the untreated control cultures, simultaneous fluorescent labeling of occludin and claudin-5 revealed continuous lines surrounding the individual cell peripheries and between cell-cell borders. Exposure to AGEs caused this labeling to become weaker and discontinuous or fractured compared with untreated control cultures, particularly in the case of occludin. When AGEs were co-administered with BiPS, however, the labeling again appeared as unbroken lines surrounding the cell peripheries and few fractures (TJ gaps) were observed. Furthermore, immunostaining was more intense compared with the cells treated with AGEs alone, indicating that the surface expression of occludin and claudin-5 was rescued by the MMP-2/9 inhibitor.

BiPS eliminates the effect of AGEs on occludin, claudin-5 and $M M P-2 / 9$. Subsequently, the association between TJ protein expression and MMP-2/9 activity in the AGE-treated cultures was examined in the presence and absence of BiPS (Fig. 5). The expression levels of occludin and claudin-5 were significantly reduced following AGE treatment, while neither occludin nor claudin-5 expression were significantly different from those in controls in cultures co-treated with AGEs and BiPS (Fig. 5A). Concomitant with the AGE-induced reduction in occludin and claudin-5, MMP-2/9 activity was elevated, while co-administration with BiPS again reversed this AGE-induced increase in MMP-2/9 activity (Fig. 5B).

\section{Discussion}

In the present study, it was demonstrated that exogenous AGEs significantly increased the permeability of GEnC monolayers, upregulated the activity of MMP-2/9 and decreased the expression of the TJ proteins occludin and claudin-5. The organic MMP-2/9 inhibitor BiPS reversed the AGE-induced hyperpermeability of GEnCs and the decrease in occludin/claudin-5 expression, suggesting that AGEs increased transendothelial permeability by increasing MMP-2/9 activity with subsequent MMP-mediated degradation of the TJs.

Hyperpermeability is a common vascular complication associated with diabetes. Several processes have been implicated in vascular pathogenesis, including the accumulation of AGEs in endothelial cells (6,7). Consistent with previous studies, it was identified that AGEs increased the permeability of GEnCs as evidenced by lower transendothelial electrical resistance and higher transendothelial FITC-dextran permeability. Immunofluorescence imaging revealed that the rows of 
TJs between the adjacent endothelial cells became fractured or discontinuous, thus creating low resistance gaps, while immunostaining also demonstrated weaker surface occludin and claudin-5 expression in the TJs following exposure. These effects were inhibited by BiPS, suggesting that AGEs disrupt TJs between the cells, at least in part, by MMP-mediated proteolysis.

Increased MMP activity can increase vascular permeability $(21,22)$; therefore, the activity of MMP-2/9 following AGE administration was examined. Indeed, the activity of MMP-9 was markedly enhanced by the AGEs and this response was dose- and time-dependent. Previously, MMP-9 was observed to decrease the expression of occludin and claudin-5, leading to an increased permeability across the blood-brain barrier and consequent retinopathy $(13,22,23)$. Therefore, MMP-9 may be an important factor regulating microvasculature permeability in numerous tissues. In addition to MMP-9, MMP-2 also increased following AGE exposure, although to a lesser extent and later compared with that of MMP-9. Although AGEs upregulated the activity of MMP-2 and MMP-9, leading to $\mathrm{TJ}$ protein degradation, whether MMP-2, MMP-9, or the two together, mediated the proteolysis of TJ protein remains to be elucidated. Bojarski et al (24) demonstrated that occludin contains a putative extracellular MMP cleavage site that may allow degradation by MMPs. However, degradation by MMPs and resistance to MMP-mediated proteolysis have been demonstrated for claudin-5 $(25,26)$; therefore, further studies are required to determine the substrate specificities of the individual MMPs on specific TJ proteins.

TJs provide the material foundation to restrict vascular permeability to molecules that can either diffuse across cell membranes or be carried across the membranes by specific membrane transporters (27-29). Thus, disruption of TJs is likely to increase vascular permeability to molecules, which are not normally transported by membrane permeability or active transport, resulting in the pathological appearance of serum constituents in urine. Occludin is one of the main components of TJs in the majority of tissues (30), while claudin-5 is only expressed in GEnCs in the kidney (31). The two proteins are critical in maintaining the endothelial barrier (32-34); therefore, the expression of occludin and claudin-5 was examined following exposure to AGEs in the presence or absence of BiPS. Immunoblotting analysis suggested that AGEs reduced the total cellular content of occludin and claudin-5, likely due to protein degradation, while expression levels in the cultures co-treated with AGEs and the MMP-2/9 inhibitor BiPS were not different from those in the untreated control cultures, again indicating that AGEs reduced the expression of occludin and claudin-5 by increasing MMP-2/9-mediated proteolysis.

In conclusion, the present study demonstrated that AGEs increased the permeability of GEnCs by an MMP-mediated disruption of intercellular TJs. The disruption of TJs was most likely mediated by degradation of the TJ proteins occludin and claudin- 5 by MMP-9, MMP-2 or the two in combination. This effect of AGEs was eliminated by an MMP-2/9 inhibitor. Accumulation of AGEs from chronic hyperglycemia may increase microvascular permeability by disrupting the TJs between cells, leading to proteinuria and other symptoms of diabetic nephropathy.

\section{Acknowledgements}

This study was supported by the National Natural Science Foundation of China (grant no. 30800408).

\section{References}

1. Bhonsle HS, Korwar AM, Chougale AD, Kote SS, Dhande NL, Shelgikar KM and Kulkarni MJ: Proteomic study reveals downregulation of apolipoprotein A1 in plasma of poorly controlled diabetes: a pilot study. Mol Med Rep 7: 495-498, 2013.

2. Tooke JE: Microvasculature in diabetes. Cardiovasc Res 32: 764-771, 1996.

3. Bonnardel-Phu E, Wautier JL, Schmidt AM, Avila C and Vicaut E: Acute modulation of albumin microvascular leakage by advanced glycation end products in microcirculation of diabetic rats in vivo. Diabetes 48: 2052-2058, 1999.

4. Svensjo E, Arfors KE, Raymond RM and Grega GJ: Morphological and physiological correlation of bradykinin-induced macromolecular efflux. Am J Physiol 236: H600-H606, 1979.

5. Bi YL, Wu MF, Lu LX, Du F, Sun XT, Tang SF and Xu GT: Functions of corneal endothelial cells do not change after uptake of superparamagnetic iron oxide nanoparticles. Mol Med Rep 7: 1767-1772, 2013.

6. Leto G, Pricci F, Amadio L, Iacobini C, Cordone S and Diaz-Horta O: Increased retinal endothelial cell monolayer permeability induced by the diabetic milieu: Role of advanced non-enzymatic glycation and polyol pathway activation. Diabetes Metab Res Rev 17: 448-458, 2001.

7. Bonnardel-Phu E, Wautier JL and Vicaut E: Advanced glycation end products are involved in microvascular permeability changes observed in microcirculation of diabetic rats in vivo. J Mal Vasc 25: 122-127, 2000.

8. Soulis-Liparota T, Cooper ME, Dunlop M and Jerums G: The relative roles of advanced glycation, oxidation and aldose reductase inhibition in the development of experimental diabetic nephropathy in the sprague-dawley rat. Diabetologia 38: 387-394, 1995.

9. Rojas A and Morales MA: Advanced glycation and endothelial functions: A link towards vascular complications in diabetes. Life Sci 76: 715-730, 2004.

10. Camici M: Renal glomerular permselectivity and vascular endothelium. Biomed Pharmacother 59: 30-47, 2005.

11. Beisswenger PJ, Makita Z, Curphey TJ, Moore LL, Jean S and Jean S: Formation of immunochemical advanced glycosylation end products precedes and correlates with early manifestations of renal and retinal disease in diabetes. Diabetes 44: 824-829, 1995.

12. Thrailkill KM, Clay Bunn R and Fowlkes JL: Matrix metalloproteinases: Their potential role in the pathogenesis of diabetic nephropathy. Endocrine 35: 1-10, 2009.

13. Giebel SJ, Menicucci G, McGuire PG and Das A: Matrix metalloproteinases in early diabetic retinopathy and their role in alteration of the blood-retinal barrier. Lab Invest 85: 597-607, 2005.

14. Death AK, Fisher EJ, McGrath KC and Yue DK: High glucose alters matrix metalloproteinase expression in two key vascular cells: Potential impact on atherosclerosis in diabetes. Atherosclerosis 168: 263-269, 2003.

15. Thrailkill KM, Bunn RC, Moreau CS, Cockrell GE, Simpson PM and Coleman HN: Matrix metalloproteinase-2 dysregulation in type 1 diabetes. Diabetes Care 30: 2321-2326, 2007.

16. Wang L, Wu J, Zhang W, Zhi Y, Wu Y, Jiang R and Yang R: Effects of aspirin on the ERK and PI3K/Akt signaling pathways in rats with acute pulmonary embolism. Mol Med Rep 8: 1465-1471, 2013.

17. Ahuja TS, Gopalani A, Davies P and Ahuja H: Matrix metalloproteinase-9 expression in renal biopsies of patients with hiv-associated nephropathy. Nephron Clin Pract 95: c100-c104, 2003.

18. Jung JY, Oh JH, Kim YK, Shin MH, Lee D and Chung JH: Acute UV irradiation increases heparin sulfate proteoglycan levels in human skin. J Korean Med Sci 27: 300-306, 2012.

19. Lauhio A, Sorsa T, Srinivas R, Stenman M, Tervahartiala T and Stenman UH: Urinary matrix metalloproteinase $-8,-9,-14$ and their regulators (try-1, try-2, tati) in patients with diabetic nephropathy. Ann Med 40: 312-320, 2008.

20. Peng H, Wang C, Ye ZC, Chen YR, Zhang J, Chen ZJ, Yu XQ and Lou TQ: How increased vegf induces glomerular hyperpermeability: A potential signaling pathway of rac1 activation. Acta Diabetol 47: 57-63, 2009. 
21. Huang W, Eum SY, András IE, Hennig B and Toborek M: PPARalpha and PPARgamma attenuate HIV-induced dysregulation of tight junction proteins by modulations of matrix metalloproteinase and proteasome activities. FASEB J 23: 1596-1606, 2009.

22. Yang Y, Estrada EY, Thompson JF, Liu W and Rosenberg GA: Matrix metalloproteinase-mediated disruption of tight junction proteins in cerebral vessels is reversed by synthetic matrix metalloproteinase inhibitor in focal ischemia in rat. J Cereb Blood Flow Metab 27: 697-709, 2007.

23. Gu Z, Cui J, Brown S, Fridman R, Mobashery S, Strongin AY and Lipton SA: A highly specific inhibitor of matrix metalloproteinase-9 rescues laminin from proteolysis and neurons from apoptosis in transient focal cerebral ischemia. J Neurosci 25: 6401-6408, 2005.

24. Bojarski C, Weiske J, Schoneberg T, Schroder W, Mankertz J, Schulzke J, Florian P, Fromm M, Tauber R and Huber O: The specific fates of tight junction proteins in apoptotic epithelial cells. J Cell Sci 117: 2097-2107, 2004.

25. Sun X, Han F, Yi J, Hou N and Cao Z: The effect of telomerase activity on vascular smooth muscle cell proliferation in type 2 diabetes in vivo and in vitro. Mol Med Rep 7: 1636-1640, 2013.

26. Liu W, Hendren J, Qin XJ, Shen J and Liu KJ: Normobaric hyperoxia attenuates early blood-brain barrier disruption by inhibiting MMP-9-mediated occludin degradation in focal cerebral ischemia. J Neurochem 108: 811-820, 2009.
27. Gonzalez-Mariscal L, Tapia R and Chamorro D: Crosstalk of tight junction components with signaling pathways. Biochim Biophys Acta 1778: 729-756, 2008

28. Anderson JM and Van Itallie CM: Physiology and function of the tight junction. Cold Spring Harb Perspect Biol 1: a002584, 2009.

29. Schneeberger EE and Lynch RD: The tight junction: A multifunctional complex. Am J Physiol Cell Physiol 286: C1213-C1228, 2004.

30. Feldman GJ, Mullin JM and Ryan MP: Occludin: Structure, function and regulation. Adv Drug Deliv Rev 57: 883-917, 2005.

31. Kiuchi-Saishin Y, Gotoh S, Furuse M, Takasuga A, Tano Y and Tsukita S: Differential expression patterns of claudins, tight junction membrane proteins, in mouse nephron segments. J Am Soc Nephrol 13: 875-886, 2002.

32. Paris L, Tonutti L, Vannini C and Bazzoni G: Structural organization of the tight junctions. Biochim Biophys Acta 1778: 646-659, 2008.

33. Harhaj NS and Antonetti DA: Regulation of tight junctions and loss of barrier function in pathophysiology. Int J Biochem Cell Biol 36: 1206-1237, 2004.

34. Xu ZC, Zhang Q and Li H: Differentiation of human hair follicle stem cells into endothelial cells induced by vascular endothelial and basic fibroblast growth factors. Mol Med Rep 9: 204-210, 2014. 